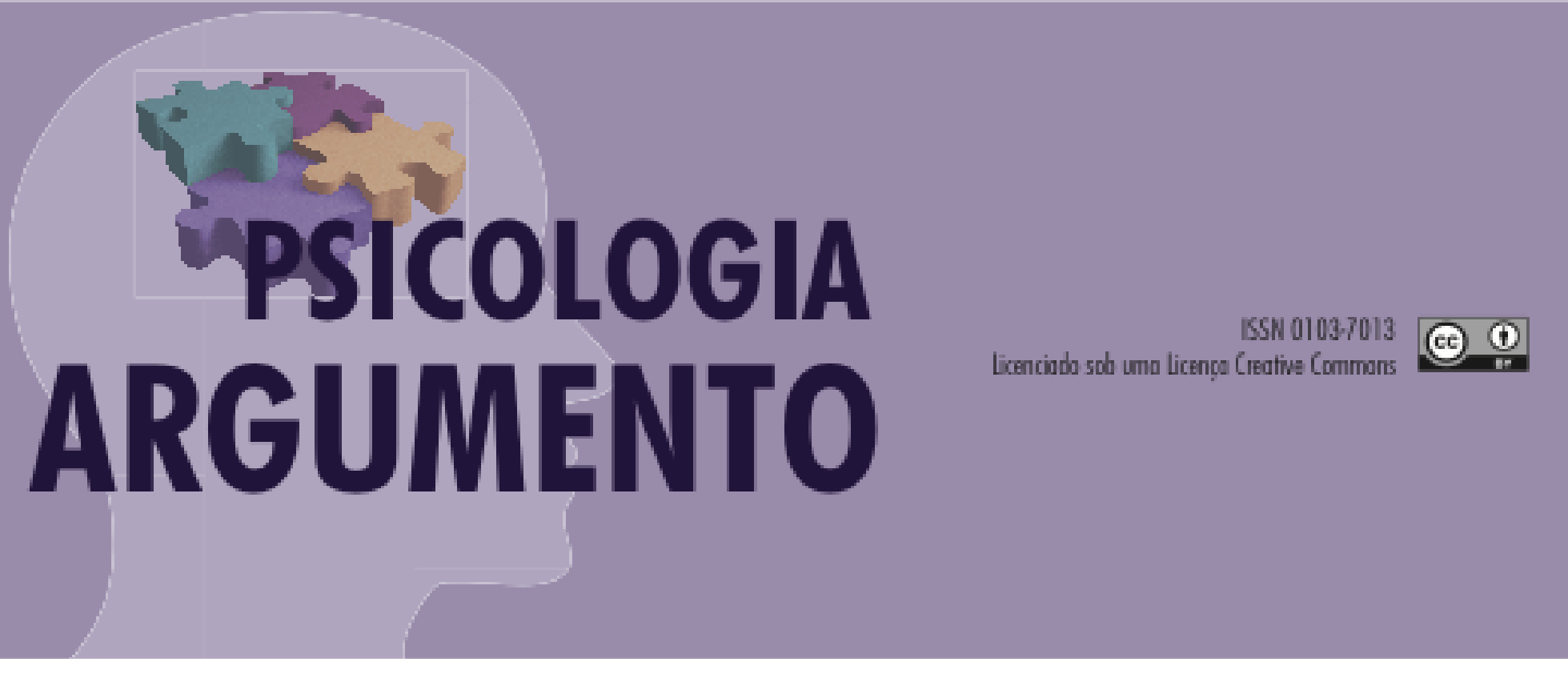

doi: http://dx.doi.org/10.7213/psicolargum.37.98.AO02

\title{
Representações sociais da hipertensão, do convívio e tratamento da doença
}

Social representations of hypertension, conviviality and treatment of the disease Representaciones sociales de hypertension, convivencia y tratamiento de la enfermedad

\section{Jean Paulo da Silva}

Doutorando do Departamento de Psicologia da Universidade Federal de Santa Catarina. Email: jeanps.silva@gmail.com.

ORCID: https://orcid.org/0000-0001-5173-1856

\section{Mariana Luíza Becker da Silva}

Doutoranda do Departamento de Psicologia da Universidade Federal de Santa Catarina. Email: marianaluiza_b@hotmail.com ORCID https://orcid.org/0000-0002-5075-982X -

\section{Andréa Barbará da Silva Bousfield}

Professora Adjunta do Departamento de Psicologia da Universidade Federal de Santa Catarina. Email: andreabs@gmail.com ORCID: http://orcid.org/0000-0002-4333-4719

\section{Resumo}

Um significativo número de óbitos ocorre devido à hipertensão arterial sistêmica (HAS). O tratamento pode possuir dificuldades de concretização devido às ideias sociais que circulam sobre a HAS. Esta pesquisa utilizou a Teoria das Representações Sociais, tendo em vista a possibilidade de conhecimento do saber social, que pode contribuir para as políticas públicas. Assim, objetivouse identificar as representações sociais da HAS, do tratamento e do convívio com a doença para 
pessoas que vivem com essa condição clínica. Foram realizadas entrevistas semiestruturadas na residência de 60 usuários do Sistema Único de Saúde na cidade de Jaraguá do Sul - SC que convivem com a HAS há no mínimo dois anos. Os dados foram submetidos à três análises hierárquicas descendentes por meio do software IRaMuTeQ. Uma em relação à $\mathrm{HAS}$, outra ao tratamento e outra sobre o convívio com a doença. Os resultados demonstraram que a representação social da hipertensão arterial, do tratamento e do convívio com a doença compartilham entre si significados centralizados no contexto prático. Este contexto é associado principalmente ao uso do medicamento, sendo pouco mencionado o controle não medicamentoso. Ressalta-se a necessidade de políticas públicas que considerem as representações sociais sobre $o$ fenômeno.

Palavras-chave: Representações sociais, hipertensão arterial, doença crônica.

\begin{abstract}
A significant number of deaths occur due to systemic arterial hypertension (SAH). The treatment may have difficulties of implementation due to the social ideas that circulate about SAH. This research used the Theory of Social Representations, in view of the possibility of knowledge, which can contribute to public policies. Thus, the objective was to identify the social representations of hypertension, treatment and living with the disease for people that living with this clinical condition. Semi-structured interviews were conducted at the residence of 60 users of the Unified Health System in the city of Jaraguá do Sul-SC, who have been living with SAH for at least two years. Data were submitted to three descending hierarchical analyzes using the IRaMuTeQ software. One in relation to hypertension, another to treatment and another about living with the disease. The results showed that the social representation of hypertension, treatment and living with the disease share meanings centered on the practical context. This context is mainly associated with the use of the drug, with little mention of non-drug control. The need for public policies that consider social representations about the phenomenon is emphasized.
\end{abstract}

Keywords: Social representation; arterial hypertension; chronic disease.

\title{
Resumen
}

Un numero significativo de muertes ocurre debido a la hipertension arterial sistemica (HAS). El tratamiento puede tener dificultades de implementación debido a las ideas sociales que circulan sobre la HAS. Esta investigación utilizó la Teoría de las Representaciones Sociales en vista de la posibilidad de conocimiento del saber social, que puede contribuir a las politicas públicas. Por lo tanto, el objetivo fue identificar las representaciones sociales de la hipertensión, el tratamiento y la vida con la enfermedad para las personas que viven con esta condición clínica. Serealizaron entrevistas semiestructuradas en la residencia de 60 usuarios del Sistema Único de Salud en la ciudad de Jaraguá do Sul - SC que han estado viviendo con HAS al menos dos años. Los datos se sometieron a tres análisis jerárquicas descendentes utilizando el software IRaMuTeQ. Uno en relación con la hipertensión, otro con el tratamiento y otro con respecto a convivencia con la enfermedad. Los resultados mostraron que la representación social de la hipertensión, el tratamiento y la convivencia con la enfermedad comparten significados centradsos en el contexto práctico. Este contexto se asocia principalmente con el uso de la droga, con poca mención del control no farmacológico. Se enfatiza la necesidade de políticas públicas que consideren las representaciones sociales sobre el fenómeno.

Palabras clave: representación social; hipertensión arterial; enfermedad crónica. 


\section{Introdução}

A hipertensão arterial sistêmica (HAS) é uma doença crônica, caracterizada por uma pressão arterial sistólica maior ou igual a $140 \mathrm{mmHg}$ e uma pressão arterial diastólica maior ou igual a $90 \mathrm{mmHg}$ (Malachias et al., 2016). Ao longo das décadas tem se desenhado um grande desafio mundial para redução das mortes e agravos associados a HAS (Stopa et al., 2018; World Health Organization, 2018). No Brasil, a HAS atinge $32,5 \%$ (36 milhões) de indivíduos, sendo mais de 60\% dos idosos, o que contribui para $50 \%$ das mortes por doença cardiovascular (Scala, Magalhães, \& Machado, 2015).

Por não possuir cura, a HAS demanda um tratamento que se prolonga por toda a vida, objetivando o controle da pressão arterial e a melhoria na qualidade de vida da pessoa. O tratamento, ou práticas de controle dessa doença silenciosa e progressiva, constitui-se no uso de medicação anti-hipertensiva e na modificação do estilo de vida e adoção de comportamentos mais saudáveis como: alimentação adequada, redução do consumo de sódio, consumo moderado de bebidas alcoólicas, abstinência do tabaco, redução de estresse psicoemocional e prática de atividades físicas (Brasil, 2013; Figueiredo \& Asakura, 2010). O tratamento medicamentoso associado à mudança de hábitos de vida, reduzem os riscos de complicações da doença (Malachias et al., 2016). Antunes (2017) aponta em sua pesquisa que pessoas que vivem com a hipertensão arterial enfatizam o uso de medicação como prática de controle principal, enquanto que os grupos dos profissionais de saúde e familiares enfatizam as mudanças de hábitos.

No contexto da aplicação de estratégias terapêuticas no controle da doença, a modificação de estilos de vida e adoção de comportamentos favoráveis à saúde aparecem como as principais dificuldades na efetivação do tratamento, tendo em vista que a adesão à terapêutica é deficitária quando se trata da necessidade de modificar padrões comportamentais, principalmente quando estabelecidos durante um longo tempo de vida (Figueiredo \& Asakura, 2010; Péres, Magna, \& Viana, 2003). De acordo com Canesqui (2015), embora as pessoas com hipertensão reconheçam o saber médico e a importância do tratamento, há uma grande dificuldade em seguir as orientações que interferem diretamente nas relações do sujeito consigo e com os outros. Jankowska-Polańska et al. (2016) apontam que a aceitação em relação à doença se relaciona positivamente com a 
adesão ao tratamento. Além disso, Torres e Santiago (2015) pontuam que a informação sobre as consequências e dos benefícios do tratamento estimulam a adesão ao tratamento.

Conforme Jodelet (2013), diante de uma doença, tende-se a explicar e a categorizar toda a experiência que se tem com ela, com o intuito de formar um conhecimento sobre a doença. Esta organização do conhecimento é feita a partir da cultura, que influencia o modo de compreensão da doença e de como tratá-la. Com isso, demonstra-se a necessidade de compreender a hipertensão arterial a partir das representações sociais elaboradas e compartilhadas pelas pessoas, possibilitando verificar sua relação com comportamentos específicos, como as práticas de controle da HAS. A partir do exposto, Leão e Silva et al. (2013) apresentam a relevância da Teoria das Representações Sociais (TRS) no estudo da HAS. Esta teoria compreende que as representações sociais são um mecanismo de valores, ideias e práticas, que têm como função permitir as pessoas controlarem e orientarem-se em seu meio social, assim como prover uma rede de significados que permitem a comunicação sem ambiguidade dentro de uma realidade contextual (Moscovici, 2010).

Segundo Jodelet (2013), as representações sociais são conhecimentos socialmente elaborados e partilhados, também referidos como senso comum, é transmitido pelas relações sociais, pela mídia e pelas comunicações interpessoais, e é construído através destas trocas, sendo que a assimilação destas informações orienta as práticas sociais. Estas práticas são sistemas de comportamentos inseridos em um contexto de implicação social ao qual tem sua funcionalidade dirigida. Para Wachelke e Camargo (2007) as influências que existem entre representações e comportamento são configuradas em uma via de mão dupla, onde convencionalmente na produção científica sobre representações sociais, o comportamento assume a forma de práticas sociais. Dessa forma, Silva e Bousfield (2016) apontam que através da TRS é possível apreender os significados subjetivos atribuídos pelas pessoas com hipertensão, além de conhecer as facilidades e dificuldades implicadas na terapêutica da HAS, para a elaboração de posterior planejamento de intervenções na formulação de políticas públicas. A partir disso, este estudo objetivou identificar as representações sociais da hipertensão arterial sistêmica, do tratamento e do convívio com a doença para pessoas que vivem com essa condição clínica. 


\section{Método}

Tratou-se de uma pesquisa de delineamento de levantamento de dados e caráter exploratório e descritivo, com corte transversal. Participaram da pesquisa 60 pessoas, sendo 37 mulheres e 23 homens, usuários do Sistema Único de Saúde - SUS de Jaraguá do Sul, interior de Santa Catarina, que vivem com hipertensão arterial. Os critérios de inclusão foram: pessoas com 40 anos ou mais, considerando a maior prevalência de HAS a partir dessa idade (Brasil, 2013); e, que vivem com a doença há no mínimo dois anos. Como critério de exclusão: usuários com diabetes e/ou algum tipo de transtorno grave ou deficiência que impossibilitasse a interação com o pesquisador e/ou que apresentassem a modalidade de hipertensão arterial secundária.

Para a coleta dos dados, realizou-se contato com as unidades da Estratégia Saúde da Família e após autorização da Secretaria Municipal de Saúde, foi solicitado às equipes de saúde um relatório dos usuários que se possuíam os critérios de elegibilidade e realizado sorteio dos participantes, que foram contatados em seguida pela equipe de saúde convidando-os a participar da pesquisa. A partir do aceite, foi realizado agendamento para realização da entrevista na casa dos participantes. $O$ instrumento consistiu em uma entrevista semiestruturada com questões que buscavam investigar as RS da HAS, do tratamento da hipertensão e de como é conviver com ela. Além de questões de caracterização da amostra. No início das entrevistas, o entrevistador se apresentou, comunicou as informações da pesquisa e os aspectos éticos presentes no Termo de Consentimento Livre e Esclarecido, sendo solicitado ao participante a sua assinatura. As entrevistas foram gravadas e apresentaram média de 49 minutos de duração.

Para a análise, os dados textuais foram submetidos ao software IRaMuTeQ (Interface de R pour analyses Multidimensionnelles de Textes et de Questionneires), que realiza análises quantitativas de dados textuais por meio de lexicografia (frequência e estatísticas básicas) e Classificação Hierárquica Descendente - CHD (Camargo \& Justo, 2013). Assim, neste estudo foram originados 3 corpus de análise nomeados: Hipertensão; Tratamento; e Convívio, sendo submetidos à CHD. Para análise da caracterização dos participantes, foi empregada estatística descritiva por meio do software SPSS (Statistical Package for the Social Science - Pacote Estatístico para Ciências Sociais), versão 17.0. 
Em relação aos aspectos éticos, o presente estudo obteve aprovação do Comitê de Ética em Pesquisa (CEP) da Universidade Federal de Santa Catarina sob o parecer $n^{\circ}$. 242.982, e consequente aprovação da Secretaria Municipal da Saúde de Jaraguá do Sul.

\section{Resultados}

A composição da amostra teve inicialmente a intenção de pareamento entre os sexos, entretanto houve dificuldade em obter participantes do sexo masculino que aceitassem responder à entrevista. Assim, participaram 37 pessoas do sexo feminino e 23 masculino. Infere-se que esse fato pode estar associado à característica de os homens buscam menos os serviços de saúde se comparados às mulheres, havendo influencia da imagem construída socialmente em relação aos papeis de gênero, em que homens associam a busca por cuidados nos serviços de saúde com fragilidade, vista esta como uma característica feminina. Em relação à idade, a média para o sexo masculino foi de 60 anos $(\mathrm{DP}=6,03)$ e do sexo feminino 55 anos e 8 meses $(\mathrm{DP}=7,40)$.

A maioria $(n=46)$ frequentou até o ensino fundamental, destes 31 não o concluíram, corroborando dados que mostram que para ambos os sexos, a prevalência do diagnóstico da HAS é maior quando menor o grau de instrução formal (Brasil, 2011). Além disso, 53 pessoas eram casadas ou moravam com companheiro(a), 4 eram viúvos(as) e 3 divorciados(as). Em relação ao tempo de vivência com o diagnóstico da HAS, a média para o sexo masculino foi de 10 anos e 6 meses $(D P=7,2)$ e feminino 12 anos $(\mathrm{DP}=8,09)$, dado obtido por meio do relato dos participantes.

O primeiro corpus a ser analisado pelo software IRaMuTeQ foi nomeado como “Hipertensão". Neste, foram encontrados 271 segmentos de textos, retendo 72,32\% do total, gerando 5 classes. A software dividiu o corpus Hipertensão em dois subcorpora, conforme a Figura 1. 


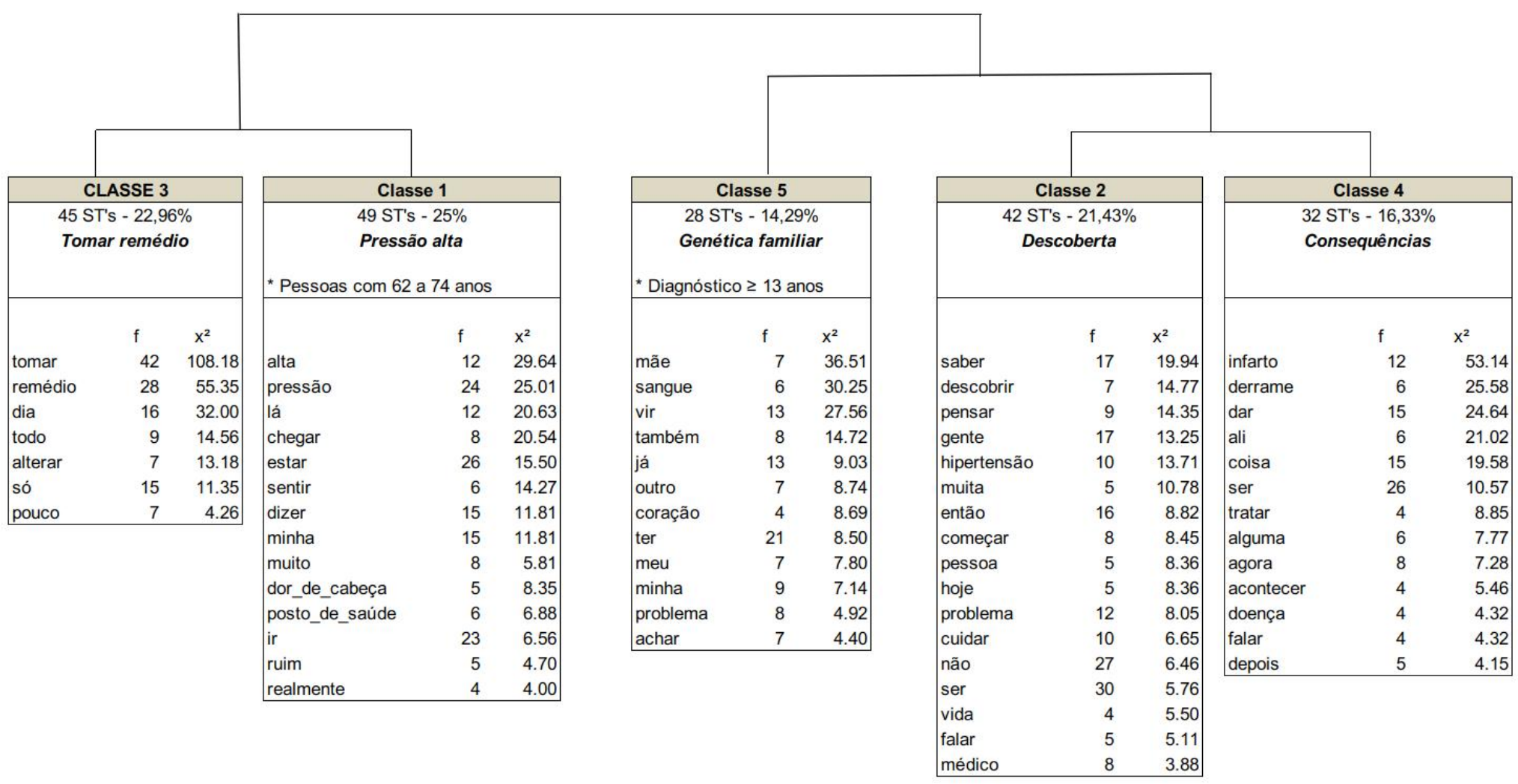

Figura 1.

Dendrograma da classificação hierárquica descendente do corpus Hipertensão.

A classe 1 intitulada "Pressão alta" foi responsável por 25\% das ST’s. Nesta classe os conteúdos estão organizados em torno dos elementos: estar; pressão; alta; dor de cabeça; e foram associados a pessoas entre 62 e 74 anos. As falas referem-se à elevação dos níveis de pressão arterial, fator que gera preocupação e medo nas pessoas que vivem com HAS, exemplificado pelo trecho: "Eu tenho muito medo, me dá bastante dor de cabeça. Eu fico desconfortável, e fico mal, fico irritada. Mais dor de cabeça" (Mulher, 48 anos, convive com a hipertensão há 5). Além disso, o surgimento de sintomas físicos como a dor de cabeça surge como motivo para que os participantes busquem assistência junto aos serviços de saúde.

A classe 3 chamada "Tomar remédio" foi responsável por 22,96\% das ST’s classificadas no corpus. Assim os principais elementos que a compõem são: tomar, remédio, dia. A HAS é percebida como a obrigação de tomar os medicamentos 
diariamente, os quais garantem o controle dos níveis pressóricos, amenizando os sintomas da doença, ilustrado pelo trecho: "Achei que estava legal já, que o tratamento estava completo, mas isso aí é contínuo, tem que tomar direto" (Homem, 64 anos, convive com a hipertensão há 6). Contudo, para eles, fatores emocionais como o estresse e a preocupação elevam os níveis de pressão arterial mesmo fazendo uso do medicamento. O "não se incomodar" surge como uma estratégia para estas pessoas controlarem a doença.

A classe 2 representa 21,43\% dos ST's classificadas e recebeu o nome de "Descoberta". Para os participantes a doença é silenciosa, em que a pessoa não sabe que possui HAS e acaba descobrindo apenas quando surgem problemas de saúde que demandam auxílio profissional, como na fala: "É um risco de vida que pode levar até à morte se não se cuida, se não procurar ir em médico ou alguma coisa. Se não se cuida, talvez você nem sabe que está com esse problema" (Mulher, 45 anos, convive com a hipertensão há 4). O profissional de saúde é citado como responsável pela descoberta da doença, após consultas motivadas por alguma complicação ou sintomas emergentes não relacionados diretamente à HAS.

A classe 4 foi intitulada “Consequências" com 16,33\% dos ST's. Os elementos destacados são: infarto, derrame, dar, coisa. A classe é representada pela ideia de que a HAS exige uma prática constante de cuidados e restrições necessárias, que visam garantir uma melhor condição de saúde. Além disso, pontuam-se as possíveis consequências que podem ocorrer caso a doença não seja descoberta a tempo, ilustrado por: "É uma coisa que pode acontecer com a gente, que pode dar infarto se estiver muito alta. A gente sente quando tem pressão alta porque sobe a dor de cabeça” (Mulher, 53 anos, convive com a hipertensão há 5).

A classe 5 chamada "Genética familiar" possui 14,29\% dos STs e foi produzida principalmente por pessoas que convivem com a hipertensão arterial há 13 anos ou mais. Seus principais elementos são: mãe, sangue, vir. Nesse sentido a doença é vista como algo que está no sangue da família, passando através das gerações, sendo este o principal causador da ocorrência da HAS. A partir disso, algumas falas pontuaram práticas de cuidados antecipados, tendo em vista a ocorrência de casos com pais e familiares, demonstrado na fala: "Eu no caso, já sofri um infarto. Ali é que eu realmente fui na 
verdade saber. Claro que eu sabia que vinha de pai, mãe, de avó. Eu na verdade até cuidava" (Homem, 64 anos, convive com a hipertensão há 16).

Analisando o dendrograma e o conteúdo obtido pela análise do corpus Hipertensão verifica-se uma noção de HAS associada de um lado ao elemento pressão alta, que possui a característica de ser um signo concreto para a doença, pois pode ser medido e elaborado enquanto algo presente, além do medicamento que controla os níveis pressóricos evitando a ocorrência dos sintomas da doença; e por outro lado à ideia de que a hipertensão é algo que vem do sangue da família, é passado através das gerações, e deve ser descoberta com rapidez para evitar complicações.

O segundo corpus analisado foi intitulado de "Tratamento" e se refere ao conteúdo originado na questão sobre o que os participantes pensam a respeito do tratamento da HAS. Assim, o software identificou 400 ST's dos quais 292 (73\%) foram considerados na CHD. Foram identificadas 1.338 palavras que ocorreram 10.628 com média de ocorrência de 7,94. O corpus foi subdividido em 4 classes distintas. Na primeira partição criou-se dois subcorpus, gerando as classes 1 e 4 e na outra as classes 2 e 3 . Na segunda partição foi separada a classe 2 da 3 e na terceira partição a classe 1 da 4 , resultando no dendograma mostrado na Figura 2.

A classe 2 chamada "Controle da pressão" foi a mais representativa do corpus com $30,82 \%$ dos segmentos de texto classificados. Os conteúdos se organizam principalmente em torno das palavras: pressão; ano; pressão alta; coração. Nesta classe o destaque principal é dado às estratégias para controle da HAS, como o uso de medicamentos, as visitas ao médico e a verificação regular da pressão arterial. A fala apresentada exemplifica o exposto: "Comprei o negócio de medir a pressão. Eu meço minha pressão em casa" (Mulher, 67 anos, convive com a hipertensão há 20).

A classe 3 "Unidade de saúde" com 16,78\% dos ST's se organiza em torno dos elementos: começar; sentir; posto de saúde; dizer. O conteúdo é representativo do sexo masculino e se refere ao contexto da unidade de saúde e ações desenvolvidas por profissionais como um elemento que compõe o tratamento, a partir de orientações, acompanhamento de medições periódicas e retirada gratuita de medicações, como na seguinte fala: "Eu já conferi no posto de saúde. Medi em casa e dai fui no posto de saúde para ver se estava certo, e estava igual, não tinha muita diferença” (Mulher, 67 anos, convive com a hipertensão há 20). 


\begin{tabular}{|c|c|c|c|c|c|c|c|c|c|c|c|}
\hline \multicolumn{3}{|c|}{ CLASSE 3} & \multicolumn{3}{|c|}{ Classe 2} & \multicolumn{3}{|c|}{ CLASSE 4} & \multicolumn{3}{|c|}{ Classe 1} \\
\hline $\begin{array}{r}49 \text { ST's } \\
\text { Unidade } \\
\text { * Sexo masculino }\end{array}$ & $\begin{array}{l}, 78 \% \\
\text { saúd }\end{array}$ & & \multicolumn{3}{|c|}{$\begin{array}{c}90 \text { ST's - } 30,82 \% \\
\text { Controle da pressão }\end{array}$} & \multicolumn{3}{|c|}{$\begin{array}{c}76 \text { ST's - } 26,03 \% \\
\text { Medicamento diário }\end{array}$} & \multicolumn{3}{|c|}{$\begin{array}{c}77 \text { ST's - } 26,37 \% \\
\text { Tratamento para viver mais }\end{array}$} \\
\hline & $f$ & $x^{2}$ & \multicolumn{3}{|r|}{$x^{2}$} & \multicolumn{3}{|c|}{$f \quad x^{2}$} & \multicolumn{3}{|c|}{ f } \\
\hline começar & 11 & 29.69 & pressão & 29 & 40.01 & tomar & 48 & 37.06 & gente & 36 & 42.33 \\
\hline sentir & 9 & 26.80 & ano & 13 & 26.54 & noite & 10 & 29.43 & tratamento & 29 & 29.59 \\
\hline pouco & 7 & 21.00 & deus & 11 & 25.66 & fácil & 11 & 21.09 & viver & 8 & 22.97 \\
\hline lá & 11 & 18.80 & minha & 20 & 21.97 & todo & 18 & 20.04 & ajuda & 7 & 15.83 \\
\hline posto_de_saúde & 10 & 16.97 & graça & 9 & 20.84 & esquecer & 8 & 19.06 & outro & 17 & 12.12 \\
\hline já & 18 & 14.41 & pressão_alta & 10 & 19.36 & dificuldade & 8 & 19.06 & doença & 6 & 10.02 \\
\hline casa & 7 & 13.39 & coração & 10 & 16.18 & dia & 21 & 13.63 & mais & 19 & 9.80 \\
\hline depois & 6 & 11.67 & estar & 49 & 14.91 & difícil & 9 & 13.19 & coisa & 21 & 9.67 \\
\hline ir & 26 & 9.64 & mês & 7 & 12.39 & achar & 20 & 11.88 & também & 14 & 8.91 \\
\hline estar & 28 & 9.14 & dar & 23 & 12.15 & nenhum & 7 & 10.40 & não & 53 & 8.72 \\
\hline ficar & 10 & 7.95 & querer & 8 & 11.74 & seguir & 6 & 10.25 & acontecer & 7 & 8.18 \\
\hline dizer & 16 & 6.46 & problema & 21 & 11.19 & ser & 55 & 9.67 & ficar & 13 & 6.42 \\
\hline então & 19 & 5.96 & dizer & 28 & 11.13 & corretamentt & 6 & 7.97 & tratar & 6 & 6.03 \\
\hline \multirow[t]{6}{*}{ ali } & 5 & 5.55 & falar & 9 & 9.41 & meu & 14 & 7.40 & conseguir & 5 & 5.53 \\
\hline & & & ter & 60 & 9.17 & tranquilo & 8 & 7.39 & mudar & 5 & 5.53 \\
\hline & & & medir & 10 & 7.97 & remédio & 32 & 6.13 & mesmo & 11 & 5.10 \\
\hline & & & nunca & 9 & 7.72 & muito & 13 & 5.91 & muita & 6 & 4.67 \\
\hline & & & ir & 40 & 6.45 & comprimido & 5 & 5.68 & & & \\
\hline & & & continuar & 5 & 3.87 & sempre & 16 & 4.18 & & & \\
\hline
\end{tabular}

Figura 2.

Dendograma da classificação hierárquica descendente do corpus Tratamento.

A classe 4 intitulada "Medicamento diário" teve 26,03\% dos ST's e os principais elementos que a compõem são: tomar; noite; fácil; todo; esquece; dificuldade. Nesta classe o tratamento está ligado à ideia de que é necessário tomar os medicamentos para a HAS todos os dias, nos horários corretos. Para os participantes essa prática é fácil quando se torna um hábito e o esquecimento é apontado quando o tratamento ainda é recente. Com o passar do tempo, as práticas medicamentosas são incorporadas às rotinas e já não são mais consideradas difíceis de serem realizadas, como o apontado em: "É coisa de rotina, é a mesma coisa que tomar um banho de noite e dormir, é coisa de rotina" (Homem, 61 anos, convive com a hipertensão há 22). 
Analisando o dendograma é possível inferir que o tratamento é colocado como a necessidade de manter a pressão arterial controlada, tendo o contexto dos serviços de saúde como possibilidades importantes de auxílio e monitoramento. Além disso, o tratamento é visto como uma prática que garante uma vida mais longeva e como maior qualidade garantida pelo uso frequente dos medicamentos anti-hipertensivos.

A última análise corresponde ao corpus chamado de "Convívio". Este corpus diz respeito à conteúdos relacionados a vivência com a hipertensão arterial. Ao ser submetido ao software, o corpus originou 488 segmentos de texto com 362 (74,18\%). Assim foram verificadas 1.499 palavras que ocorreram 12.756 vezes com média de ocorrência de 8,5 vezes. Conforme a Figura 3, a análise evidenciou 3 classes de conteúdos dividindo em um primeiro momento o corpus em dois subcorpus onde a classe 2 se diferenciou das classes 1 e 3. E em um segundo momento houve a diferenciação entre as classes 1 e 3.

A classe 3 "Medir a pressão" é a mais representativa, com 37,3\% dos ST's. Os elementos destacados foram: pressão; chegar; vez; estar; alta. Os conteúdos desta classe demonstraram que o convívio com a HAS está associado aos cuidados frequentes com a doença. Nesse sentido, a busca pelo controle faz com que práticas de cuidado sejam incorporadas ao cotidiano, sendo a medição dos níveis pressóricos a principal delas. Os serviços de saúde aparecem como principais locais onde as medições são realizadas e aonde há a descoberta da condição clínica, exemplificado em: "Eu descobri por causa da minha coluna. Eu fiquei ruim e me levaram para o hospital, ai foram ver e a pressão estava 22 por 18” (Homem, 63 anos, convive com a hipertensão há 13).

A classe 1 nomeada de "Uso prolongado de remédio" possui $29,6 \%$ dos ST's. As palavras mais frequentes foram: ano; remédio; fazer; tomar; médico. Viver com a HAS relaciona-se ao fato de a descoberta da doença marcar o início do uso de remédios por longos períodos de tempo. O remédio aparece como elemento cotidiano, que a partir do diagnóstico da doença passa a integrar as práticas diárias, ilustrado pelo trecho: “ $A i$ normalizou, 12 anos que fiz as primeiras consultas para descobrir que eu tinha pressão alta. 12 anos que eu tomo já e nunca mais parei de tomar" (Mulher, 61 anos, convive com a hipertensão há 12). Apesar de o uso do medicamento ser relatado como um hábito que se integrou à vida cotidiana, por vezes o diagnóstico da doença não determina o início imediato e adequado do uso do medicamento. Nesse sentido, muitas vezes a medicação é incluída na rotina apenas quando surge algum sintoma aparente. 


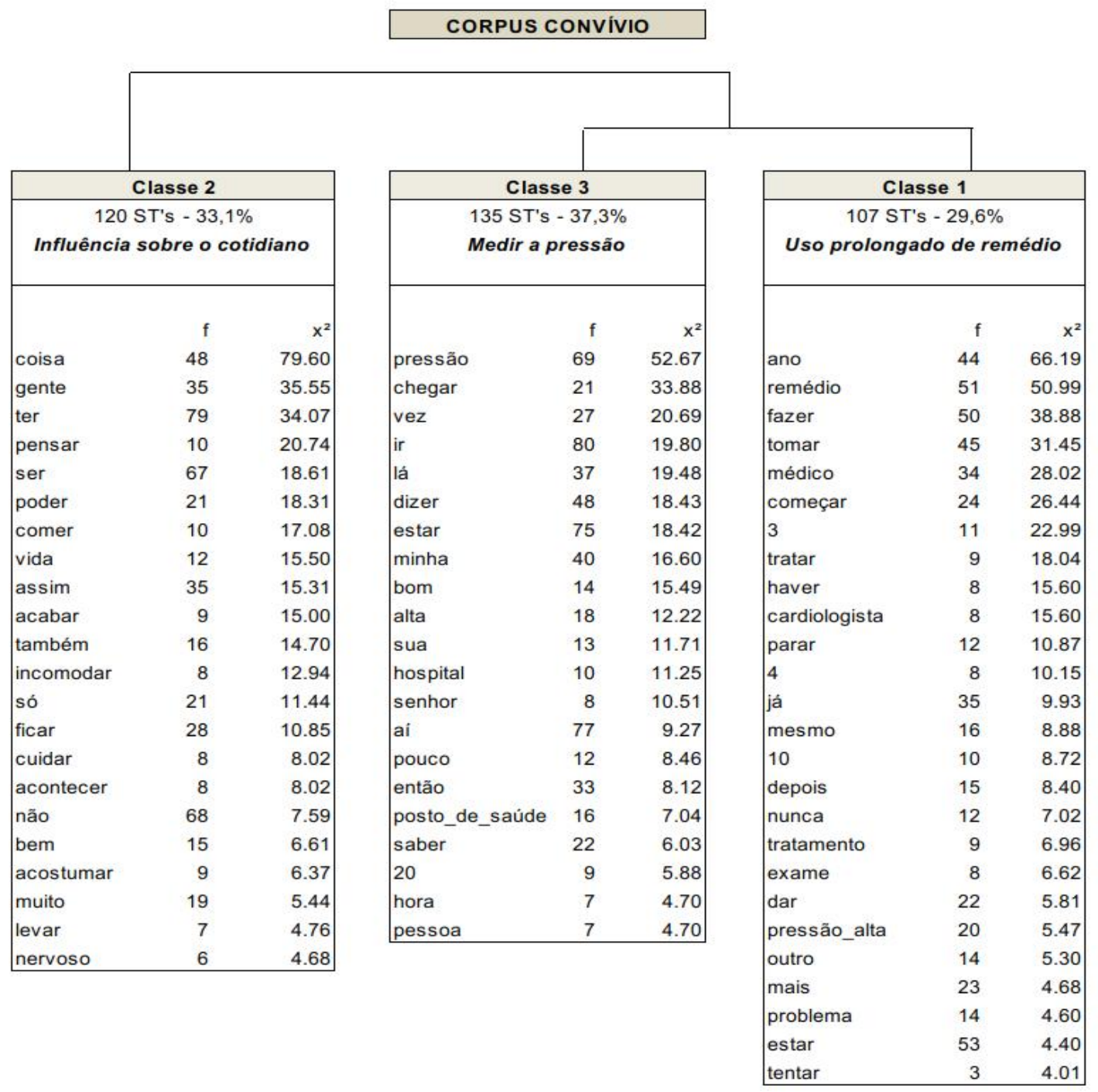

\section{Figura 3.}

Dendrograma da Classificação Hierárquica Descendente do corpus Convívio.

A classe 2 "Influência sobre o cotidiano" com 33,1\% dos ST's possui como principais elementos: coisa; gente; ter; pensar; poder; comer. Aqui, viver com a HAS é sofrer a influência que a doença exerce sobre a vida cotidiana, demandando a adoção de novas práticas e modificação de hábitos de vida, exemplificado em: "Eu tive que melhorar na minha alimentação, atividade física, todas essas coisas porque senão não adianta só tomar remédio se você não se ajuda" (Mulher, 45 anos, convive com a hipertensão há 4). 
O contexto que envolve a convivência com a HAS demonstra que a doença demanda práticas específicas, sendo a principal delas o controle constante da pressão arterial. Essa prática se relaciona com o uso prolongado de medicamentos, e este por sua vez, associado às outras práticas necessárias exerce influência sobre o cotidiano das pessoas, exigindo mudanças de hábitos de vida.

\section{Discussão}

Ao analisar os resultados apresentados observa-se que as ideias associadas à HAS se relacionam à doença em aspectos concretos. Assim, a presença constante do termo "pressão alta" assume o emblema de uma modificação no organismo da pessoa que vive com HAS. Mantovani et al. (2008) afirmam que o conteúdo imagético da hipertensão e o reconhecimento da doença está associado a uma mudança no corpo, dessa forma, quando o sintoma se manifesta e é sentido pela pessoa, o termo "pressão alta" é materializado como um representante da HAS. Na pesquisa de Silva, Bousfield e Cardoso (2013), foi constatado que o conteúdo informacional da HAS, veiculado pela mídia impressa no país, estabelecia forte ligação entre a HAS e o termo pressão alta, e este direcionado às práticas sociais que envolvem a doença.

Para os participantes da pesquisa, a descoberta da HAS geralmente é tardia em decorrência de algum sintoma ou em consultas realizadas devido a outras enfermidades. Com isso, consideram a doença como silenciosa. Nesse sentido, Leão e Silva et al. (2013) destacam em sua pesquisa, que quando os pacientes representavam a doença como silenciosa, apresentavam baixos índices de adesão ao tratamento, e quando representavam a doença como sintomática, a partir de sintomas percebidos, apresentavam maior adesão. Em contrapartida, Silva e Bousfield (2016) observaram que apesar de alguns participantes não possuírem sintomas físicos aparentes, estes se preocupavam e aderiam ao tratamento igualmente em decorrência da representação da HAS ser silenciosa e perigosa. Além disso, Bezerra, Lopes e Barros (2014) revelam a importância da aceitação da doença no desenvolvimento de ações de cuidado. Assim, o reconhecimento da doença e sua representação influenciam as práticas de controle, mas parecem ser influenciadas por mais variáveis pertinentes à contexto e vivência. 
Além disso, a descoberta se relacionou com as consequências desta condição. Péres, Magna e Viana (2003) referem que a possibilidade de ocorrência dessas complicações acarreta medo quando os pacientes percebem que estão com a pressão alta. Nesta pesquisa, os participantes relataram que quando ficam preocupados com alguma situação pessoal, a pressão arterial aumenta, mesmo com o tratamento. Isso vai ao encontro do descrito em outros documentos (Brasil, 2013; Malachias et al., 2016; Weber et al., 2014) ao indicar que o fator emocional é uma variável importante para a HAS. Ademais, alguns participantes apresentaram a ideia de que a HAS é determinada geneticamente, assim, percebem a importância da antecipação de cuidados de saúde quando se tem casos de HAS na família.

Em relação ao tratamento da HAS, verifica-se que as representações sociais são intimamente atreladas à representação da própria doença em si. O conteúdo representacional do tratamento se relaciona com o contexto prático que envolve a doença. Assim, a demanda cotidiana do seu controle é o principal elemento do objeto representacional. Em conformidade, Silva e Bousfield (2016) e Antunes (2017) encontraram que as representações sociais do tratamento da HAS são sobrepostas às representações sociais da própria doença, assim, práticas de cuidado e a doença fazem parte de um mesmo campo representacional interdependente.

De forma geral, para os participantes o tratamento é concretizado em práticas como medir a pressão com frequência, ir a consultas médicas e tomar os medicamentos. Outras práticas de controle que envolvem mudanças de hábitos foram pouco mencionadas, sendo a prática medicamentosa central na representação do tratamento. Isso demonstra a medicalização como fenômeno social presente na atualidade, onde a busca por curas rápidas e com menor ônus injeta no medicamento as expectativas dos pacientes (Morin, 2005). A centralidade da medicação é relacionada com o contexto social da doença e pelo fato de haver resistência e dificuldades na adoção de mudanças de hábito considerados mais saudáveis e nem sempre prazerosos em longo prazo (Antunes, 2017; Stuhler, 2012). As mudanças de estilo de vida exigem modificações em práticas sociais já estabelecidas, representando uma mudança na vida diária para o resto da vida, tendo em vista a cronicidade da HAS. Com isso, há uma associação de sentimentos negativos e o tratamento não medicamentoso se torna uma dificuldade na efetivação do tratamento (Silva \& Bousfield, 2016). Nesse sentido, as restrições alimentares, de bebida, de cigarro, 
o apelo para a realização de atividades físicas regulares, os afastamentos do trabalho, são fatores que impactam o cotidiano de quem convive com a HAS (Canesqui, 2015).

O tratamento é considerado necessário e o compromisso diário com os medicamentos é a contrapartida para uma vida de maior qualidade e longevidade. Para os participantes, o uso correto do medicamento é encarado inicialmente como difícil, contudo, com o tempo ele é incorporado à rotina e avaliado como algo de fácil realização. Esses achados diferem dos encontrados por Figueiredo e Asakura (2010) onde tomar os remédios diariamente foi considerado difícil, devido à outras tarefas do cotidiano e esquecimentos dos horários corretos. Já na pesquisa de Silva e Bousfield (2016), as autoras encontraram que o tratamento medicamentoso aparece como uma facilidade para alguns, tornando-se um hábito, e já para outros participantes há a associação de um sentimento negativo à medicação, devido o fato desta prática ter que ser realizada pelo resto da vida (Silva \& Bousfield, 2016).

Para quase toda a amostra estudada $(n=57)$ a HAS é um objeto de interesse, conversam com familiares e amigos sobre o assunto e trocam informações a respeito do tratamento. Wagner (1998) advoga que a representação de um objeto deve ser relevante para o grupo de referência. Assim, não há representação social se o objeto não possui uma implicação para as pessoas. Além disso, a maioria dos participantes afirmou que os profissionais de saúde são a principal fonte de informação a respeito da HAS, sendo também um local onde são validadas as informações relacionadas ao tratamento. Stuhler (2012) ao estudar o diabetes - doença crônica semelhante - verificou que os profissionais de saúde também foram citados como principal fonte de informação. Essa condição otimiza as possibilidades de uma comunicação educativa entre os pacientes e a equipe profissional.

Para isso, Bousfield e Camargo (2011) pontuam a necessidade de uma comunicação adequada entre equipe e paciente, pois é no processo de interação que ocorre a adoção de modelos cognitivos, determinação de tarefas e criação de representações. Nessa direção, Almeida, Paz e Silva (2012) afirmam que compete às equipes de saúde desenvolver ações mais abrangentes que abarquem os diversos saberes para o cuidado com a HAS. Em geral, os participantes avaliaram positivamente a relação com as equipes de saúde. Dias, Souza e Mishima (2016) afirmam que o vínculo com os profissionais de saúde e o seu cuidado humanizado facilitam a adesão ao tratamento. 
Em relação ao convívio com a HAS novamente há uma centralidade das práticas de controle da doença. Assim o elemento "medir a pressão arterial" figura como um símbolo do que é conviver com a HAS, inserindo o indivíduo no espaço físico e relacional da unidade de saúde, que é referida como o principal local de realização dessa prática. $O$ uso prolongado do medicamento se constitui em outra prática da convivência com a doença que influencia o cotidiano, assim como a necessidade de mudança de hábitos. Para Silva, Budó, Silveira, Badke e Beuter (2013) a HAS exige uma reaprendizagem corporal, envolvendo ações de proteção do corpo não realizadas anteriormente, passando a aderir a hábitos que influenciam diretamente a vida cotidiana.

\section{Considerações Finais}

Esta pesquisa pretendeu identificar as representações sociais da hipertensão arterial sistêmica, do tratamento e do convívio com a doença para pessoas que vivem com essa condição crônica. A partir dos conteúdos apresentados, verifica-se uma representação social da HAS ligada a uma mudança no organismo que tem a sua materialização nos sintomas que surgem com ela, apesar de também ser percebida como silenciosa, tendo em vista que nem sempre apresenta sintomas.

Ao se referirem a pressão alta os participantes evocam as práticas que a acompanham, centralizadas principalmente no uso de medicamentos, os quais possuem compromisso diário a fim de evitar consequências como infarto e derrame. As práticas não medicamentosas são pouco mencionadas, retratando a dificuldade da mudança de hábitos no cotidiano dos pacientes. Pontua-se a necessidade de implementar ações baseadas na educação em saúde que potencializem a realização de práticas de controle não medicamentosas. Os comportamentos de controle da doença são alicerçados e validados pelo conhecimento médico, sendo os serviços de saúde um local importante para os participantes. Aponta-se a importância de um cuidado em saúde que reconheça o saber social e as representações sociais da HAS.

Em geral, constata-se que ao representar a doença, os participantes lançam mão também do tratamento para organizar os conhecimentos que possuem da HAS. Assim, há um entrelaçamento entre as representações sociais da HAS, do tratamento e do convívio com a doença. Assim, as um sistema de representações se estabelece entre as três 
temáticas propostas, sendo que o convívio com a doença está associado às práticas relacionadas ao seu controle e que por sua vez estão atreladas à representação da própria doença.

Dentre as limitações da pesquisa, pontua-se o número de participantes; a ausência de uma questão para investigar se os participantes estavam com a HAS controlada, a partir da medição direta, sendo esta informação importante para o entendimento dos dados obtidos; e, o fato da Unidade de Saúde ter intermediado o contato, pode ter contribuído para respostas socialmente esperadas, mesmo sendo esclarecido não se tratar de uma pesquisa vinculada à Unidade de Saúde. Por fim, compreende-se que a temática é relevante em decorrência do aumento de número de diagnósticos de HAS e a compreensão do saber social relacionado a esse campo pode contribuir para políticas públicas de qualidade. Assim, considera-se relevante novos estudos da temática.

\section{Referências}

Almeida, G. B. S., Paz, E. P. A., \& Silva, G. A. (2012). Representações sociais de portadores de hipertensão arterial sobre a doença: o discurso do sujeito coletivo. Revista Mineira de Enfermagem, 17(1), 459-465.doi: 10.1590/S010321002011000400003

Antunes, L. (2017). Representações sociais da hipertensão arterial e do tratamento para profissionais de saúde, pessoas que vivem com hipertensão e seus familiares. Tese apresentada no Programa de Pós Graduação de Psicologia na Universidade Federal de Santa Catarina. Florianópolis/SC.

Araújo, J. C., \& Guimarães, A. C. (2007). Controle da hipertensão arterial em uma unidade de saúde da família. Revista de Saúde Pública, 41(3), 368-374. doi: $10.1590 / \mathrm{S} 0034-89102007000300007$

Bezerra, S. M. A., Lopes, L. J., \& Barros, A. L. B. L. (2014). Adesão de pacientes hipertensos ao tratamento medicamentoso. Revista Brasileira de Enfermagem, 67 (4): 550-555.doi: 10.1590/0034-7167.2014670408

Bousfield, A. B., \& Camargo, B. V. (2011). Divulgação do conhecimento científico sobre aids e representações sociais. Acta Colombiana de Psicologia, 14, 31-45. Recuperado de http://www.scielo.org.co/pdf/acp/v14n1/v14n1a04.pdf 
Brasil. Ministério da Saúde. Secretaria de Atenção à Saúde. (2013). Estratégias para o cuidado da pessoa com doença crônica: hipertensão arterial sistêmica.Brasília: $\begin{array}{llll}\text { Ministério da } & \text { Saúde. }\end{array}$ http://189.28.128.100/dab/docs/portaldab/publicacoes/caderno_37.pdf

Brasil. Ministério da Saúde. Secretaria de Vigilância em Saúde. Departamento de Análise de Situação de Saúde. (2011). Plano de ações estratégicas para o enfrentamento de doenças crônicas não transmissíveis (DCNT) no Brasil 2011-2022. Brasília: Ministério da Saúde. Recuperado de http://bvsms.saude.gov.br/bvs/publicacoes/plano_acoes_enfrent_dent_2011.pdf

Camargo. B. V., \& Justo, A. M. (2013). IRaMuTeQ: Um software gratuito para análise de dados textuais. Temas em Psicologia, 21(2), 513-518.doi: 10.9788/TP2013.2-16

Canesqui, A. M. (2015). "Pressão alta” no cotidiano: representações e experiências. Rio de Janeiro: Editora Fiocruz.

Dias, E. G., Souza, E. L. S., \& Mishima, S. M. (2016). Influência da enfermagem na adesão do idoso ao tratamento da hipertensão. Rev. Gest.Saúde (Brasília), 7(3): 115672. Recuperado de https://periodicos.unb.br/index.php/rgs/article/view/3625

Figueiredo, N. N., \& Asakura, L. (2010). Adesão ao tratamento anti-hipertensivo: dificuldades relatadas por indivíduos hipertensos. Acta Paulista de Enfermagem, 23(6), 782-787.doi: 10.1590/S0103-21002010000600011

Jankowska-Polańska, B., Blicharska, K., Uchmanowicz, I., \& Morisky, D. E. (2016). The influence of illness acceptance on the adherence to pharmacological and nonpharmacological therapy in patients with hypertension. European Journal of Cardiovascular Nursing, 15(7), 559-568.doi: 10.1177/1474515115626878.

Jodelet, D. (2013). Culture and health practices. In: A.S. De Rosa. (org). Social representations in the social arena. (pp. 153-165). New York: Routledge.

Leão e Silva, L. O., Dias, C. A., Rodrigues, S. M., Soares, M. M., Oliveira, M. A., \& Machado, C. J. (2013). Hipertensão Arterial Sistêmica: Representações Sociais de idosos sobre a doença e seu tratamento. Cadernos Saúde Coletiva, 21(2), 121-128.doi: 10.1590/S1414-462X2013000200004

Malachias, M. V. B., et al. (2016). $7^{\text {a }}$ Diretriz Brasileira de Hipertensão Arterial. Arquivos Brasileiros de Cardiologia, 107(3Supl.3), 1-83.doi: 10.5935/abc.20160152 
Mantovani, M. F., Ulbrich, E. M., Pinotti, S., Giacomozzi, L. M., Labronici, L. M., \& Sarquis, L. M. M. (2008). O significado e a representação da doença crônica: conhecimento do portador de hipertensão arterial acerca de sua enfermidade. Cogitare Enfermagem, 13(3), 336-342.doi: 10.5380/ce.v13i3

Morin, M. (2005). Atenção à doença e relação de ajuda: crise das práticas e conflitos de representação. In: Oliveira, D. C., Campos, P. H. Representações sociais: uma teoria sem fronteiras. (pp. 109-118). Rio de Janeiro: Museu da República.

Moscovici, S. (2010). Representações Sociais: investigações em psicologia Social. Petrópolis: Vozes.

Péres, D. S., Magna, J. M., \& Viana, L. A. (2003). Portador de hipertensão arterial: atitudes, crenças, percepções, pensamentos e práticas. Revista de Saúde Pública. 37(5), 635-642.doi: 10.1590/S0034-89102003000500014

Scala, L. C, Magalhães, L. B., \& Machado, A. (2015). Epidemiologia da hipertensão arterial sistêmica. In: S. M. Moreira \& A.V. Paola. Sociedade Brasileira de Cardiologia. Livro Texto da Sociedade Brasileira de Cardiologia. 2a . edição. (pp. 780785). São Paulo: Manole.

Silva, F. M., Budó, M. L. D., Silveira, C. L., Badke, M. R., \& Beuter, M. (2013). Hipertensão: condição de não doença - o significado da cronicidade na perspectiva dos sujeitos. Texto \& Contexto - Enfermagem, 22(1), 123-131.doi: 10.1590/S010407072013000100015

Silva, J. P., Bousfield, A. B. S., \& Cardoso, L. H. (2013). A hipertensão arterial na mídia impressa: análise da revista Veja. Psicologia e Saber Social, 2(2). 191-203. doi:10.12957/psi.saber.soc.2013.8793

Silva, M. L. B., \& Bousfield, A. B. S. (2016). Representações sociais da hipertensão arterial. Temas em psicologia, 24(3), 895-909.doi: 10.9788/TP2016.3-07.

Stopa, S. R., Cesar, C. L. G., Segri, N. J., Alves, M. B. A., Barros, M. B. A., \& Goldbaum, M. (2018). Prevalência da hipertensão arterial, do diabetes mellitus e da adesão às medidas comportamentais no município de São Paulo, Brasil, 2003-2015. Cad. Saúde Pública 34(10), [online].doi: 10.1590/0102-311x00198717 
Stuhler, G. D. (2012). Representações sociais e adesão ao tratamento do diabetes mellitus tipo 2. Tese de Doutorado. Programa de Pós-Graduação em Psicologia, Universidade Federal de Santa Catarina, Florianópolis.

Torres, G. M. C., \& Santiago, E. S. (2015). Adesão ao tratamento em pessoas com $\begin{array}{llll}\text { hipertensão } & \text { arterial. } & \text { REFACS, } & \text { 3(3):189-193. }\end{array}$ https://doi.org/10.18554/refacs.v3i3.1236

Wagner, W. (1998). Sócio-gênese e características das representações sociais. In A. S. P. Moreira, \& D. C. de Oliveira. (Eds.), Estudos interdisciplinares de representação social (pp. 3-25). Goiânia: AB.

Wachelke, J. F. R., \& Camargo, B. V. (2007). Representações sociais, representações individuais e comportamento. Revista Interamericana de Psicologia, 41(2), 379-390. Recuperado de http://pepsic.bvsalud.org/pdf/rip/v41n3/v41n3a13.pdf

Weber, M. A., Schiffrin, E. L., White, W. B., Mann, S., Lindholm, L. H., Kenerson, J. G., ... Harrap, S. B. (2014). Clinical Practice Guidelines for the Management of Hypertension in the Community: A Statement by the American Society of Hypertension and the International Society of Hypertension Clinical Practice Guidelines for the Management of Hypertension in the Comm. Journal of Clinical Hypertension, 16(1), 14-26.doi: 10.1111/jch.12237

World Health Organization. (2018). Noncommunicable diseases: contry profiles 2018, Geneva: World Health Organization. Recuperado de: https://www.who.int/nmh/publications/ncd-profiles-2018/en/ 